St. Mark's electrode used in the study has the distance between the stimuli and recording sites known in a region of difficult access for measuring. This would become the method more standardized and appropriate for pelvic floor, besides probably causing less discomfort than the transmuscular perineal route. These alternative methods for PNTML assessment might be useful in routine practice, mainly for intraoperative monitoring. Nevertheless it should be tested in further studies.

\section{Reference}

1. Lefaucheur JP, Yiou R, Thomas C: Pudendal nerve terminal motor latency: age effects and technical considerations. Clin Neurophysiol. 2001; 112: 4726.

\title{
Re: Percutaneous Nephrolithotomy with and without Retrograde Pyelography: A Randomized Clinical Trial
}

\author{
Ali Tabibi, Hamed Akhavizadegan, Kia Nouri-Mahdavi, Mohammad Najafi- Semnani, \\ Mojgan Karbakhsh, Ali R. Niroomand
}

Section of Urology, Dr Labbafinejad Hospital, Shahid Beheshti University of Medical Sciences, Tehran, Iran, Section of Urology, Baharloo Hospital (HA) and Department of Community Medicine (MK), School of Medicine, University of Tehran, Tehran, Iran

Int Braz J Urol, 33: 19-24, 2007

To the Editor:

This article by Tabibi et al. evaluates whether there is a benefit to performing cystoscopic ureteral catheter placement, for retrograde opacification of the renal pelvis and calyces, in order to obtain access prior to percutaneous nephrolithotomy (PCNL). The study was randomized to either no catheter placement (intervention group) or catheter placement (control group). No statistical differences were found when comparing demographic or intraoperative parameters (surgical duration, radiation exposure, hospital stay, postoperative fever) between the 2 groups. Interestingly, postoperative hemoglobin decrease was found to be significantly higher in the catheterized group ( $\mathrm{p}<0.001)$. While no statistical difference was identified for outcome $(\mathrm{p}=0.136)$, a greater percentage of patients were found to be "stone free" on KUB in the catheterized group than the noncatheterized group, $93 \%$ vs. $79 \%$, respectively. Based on these results, it would appear that while retrograde placement of a ureteral catheter does not influence the safety or duration of the procedure, it may facilitate the efficacy of PCNL in terms of achieving stone free status.

The decision for selecting a noncatheterized versus a catheterized approach should take into account the experience of the individual obtaining access as well as the clinical scenario in terms of stone location and burden. In many institutions, initial access is obtained by an experienced interventional radiologist followed by PCNL that is performed by the urologist. Even in this circumstance, it is not uncommon for the interventionalist to request a ureteral catheter for retrograde opacification of the 
collecting system. Early in one's experience, a catheterized approach may be beneficial for maximizing successful access with minimal complications. Once a level of comfort with percutaneous anatomy is achieved, a noncatheterized approach may be a natural evolution to performing percutaneous nephrolithotomy.

A larger randomized study would assist in determining the value of pre-procedure catheter placement for obtaining optimal access to the collecting system. As there are a limited number of published randomized studies on access relating to percutaneous nephrolithotomy, the authors should be congratulated on their contribution."

Dr. Ravi Munver

Chief, Minimally Invasive Urologic Surgery Hackensack University Medical Center Hackensack, New Jersey, USA E-mail:rmunver@humed.com

\title{
Re: Antibiotic Resistance and Trend of Urinary Pathogens in General Outpatients from a Major Urban City
}

\author{
Carlos R. Kiffer, Caio Mendes, Carmen P. Oplustil, Jorge L. Sampaio
}

Section of Microbiology, Fleury Institute, Sao Paulo, SP, Brazil

Int Braz J Urol, 33: 42-49, 2007

To the Editor:

Investigations of antibiotics susceptibility profiles on urinary tract infections in outpatients in São Paulo, Brazil, were aimed to recommend the ideal empirical therapeutic recipe in suspected cases of urinary tract infections (1). That had been a commendable exercise through for the maximal utility of any retrospective data in clinical practice, it would have been better to evaluate identical profiles in hospitalized patients as well. Clinicians would be treating patients during and after hospitalization. A watch is being continued on combined susceptibility pattern in a private, tertiary care hospital in the Indian capital metropolis.

Effective October 2004, susceptibility data was compiled at Sant Parmanand Hospital, a 140- bedded tertiary care hospital catering to population in the capital and adjoining townships. The antimicrobial susceptibility on urinary isolates would be determined using disk diffusion method for amoxicillin-clavulanic acid, ampicillin-sulbactam, cefaclor, cefuroxime, ceftriaxone, ceftazidime, cefotaxime, ceftizoxime, amikacin, gentamicin, netilmicin, ciprofloxacin, ofloxacin, pefloxacin, norfloxacin, nalidixic acid, chloramphenicol and nitrofurantoin. The outstanding antimicrobials were selected based on the previous quarterly susceptibility pattern, from October to December 2004. Based on the picture in 130 isolates namely, E. coli, 103, Klebsiella species, 20, Proteus species, 13 and Pseudomonas species 11, data was computed to 